\title{
Theorems on list-coloring
}

\author{
Dharmaiah Gurram ${ }^{1}$, N.Vedavathi ${ }^{1}$, \\ 1.Asst.Professor in Mathematics,K L University,Guntur (dist)A.P,India-522502
}

\begin{abstract}
Graph coloring is a well-known and well-studied area of graph theory with many applications. In this paper, we will consider two generalizations of graph coloring. In particular, list-coloring and sum-list-coloring.
\end{abstract}

Key Words: plane graph, planar graph, 2-connected.

\subsection{DEFINITIONS}

\section{INTRODUCTION}

In this paper, we assume a general knowledge of basic graph theory terminology and results.

For a vertex $\mathrm{v}$ and a set of vertices $\mathrm{X}$, we write $\mathrm{v} \sim \mathrm{X}$ if $\mathrm{v}$ is adjacent to all vertices in $\mathrm{X}$.

Definition 1.1. For two vertices $x$ and $y$ in a graph $G$, the distance dist $(x, y)$ is the length of, or number of edges in, a shortest path between them.

Definition 1.2. For a subset $P$ of vertices in a graph $G$, the $\operatorname{distance} \operatorname{dist}(P)=\operatorname{dist}(P, G)$ is defined as $\operatorname{dist}(\mathrm{P}):=\min \operatorname{dist}(\mathrm{x}, \mathrm{y})$.

$$
\operatorname{dist}(\mathrm{P}):=\min _{x, y \in P} \operatorname{dist}(\mathrm{x}, \mathrm{y})
$$

For any vertex $\mathrm{v} \in \mathrm{V}(\mathrm{G})$, let $\mathrm{N}(\mathrm{v})=\mathrm{N}(\mathrm{v}, \mathrm{G})$ denote the neighborhood of $\mathrm{v}$ in $\mathrm{G}$. In other words, $\mathrm{N}(\mathrm{v}, \mathrm{G})=\{\mathrm{u} V(\mathrm{G}): \mathrm{u} \sim \mathrm{v}\}$. This definition can also be restricted to the neighborhood of $\mathrm{v}$ in a subgraph of $\mathrm{G}$. When the graph $\mathrm{G}$ is implied, the subscript $\mathrm{G}$ will often be omitted. For a vertex set $X$ in $G$, let $N(X)=N(G[X])$ be the set of neighbors of vertices from $X$ not in $X$.

Let $\mathrm{H}$ be a subgraph of $\mathrm{G}$ and $\mathrm{c}$ be a vertex coloring of $\mathrm{H}$. For $\mathrm{v} \notin \mathrm{V}(\mathrm{H})$, let $\mathrm{c}(\mathrm{v}, \mathrm{H})=\{\mathrm{c}(\mathrm{u}): \mathrm{u} \quad \mathrm{N}(\mathrm{v}) \mathrm{n} \mathrm{V}(\mathrm{H})\}$ be the set of colors used on neighbors of $\mathrm{v}$ in $\mathrm{H}$.

For an induced subgraph $\mathrm{H}$ of $\mathrm{G}$ and $\mathrm{v} \notin \mathrm{V}(\mathrm{G})-\mathrm{V}(\mathrm{H})$, let $\mathrm{L}_{\mathrm{c}}(\mathrm{v}, \mathrm{H})=\mathrm{L}(\mathrm{v})-\mathrm{c}(\mathrm{v}, \mathrm{H})$. When the subgraph $\mathrm{H}$ is clear, we use $\mathrm{L}_{\mathrm{c}}(\mathrm{v})$. We say $\mathrm{H}$ is colored nicely by a coloring $\mathrm{c}$ with respect to lists $\mathrm{L}$ if $\mathrm{c}$ is an L-coloring of $\mathrm{H}$ and for every vertex $v \in N(H), \quad\left|\mathrm{L}_{\mathrm{c}}(\mathrm{v}, \mathrm{H})\right| \geq 3$. We also say $\mathrm{c}$ is a nice coloring of $\mathrm{H}$ in this case.

Let $\mathrm{H}$ be a proper induced subgraph of $\mathrm{G}$. For a vertex $\mathrm{v} \in \mathrm{V}(\mathrm{G}-\mathrm{H})$, let $\mathrm{d}(\mathrm{v}, \mathrm{H}):=$

$|N(v) \cap v(H)|$ be the size of the neighborhood of $v$ in $H$. Similarly, for a vertex $v \quad V(H)$, let d $(v, G-H):=\mid N$ (v) $n \mathrm{~V}(\mathrm{G}-\mathrm{H}) \mid$ be the size of the neighborhood of $\mathrm{v}$ outside of $\mathrm{H}$.

A vertex from $\mathrm{N}(\mathrm{H})$ adjacent to at least three vertices in $\mathrm{H}$ is called a three-neighbor, or simply 3-neighbor, of $\mathrm{H}$. We denote the set of 3-neighbors of $\mathrm{H}$ by $\mathrm{N}$

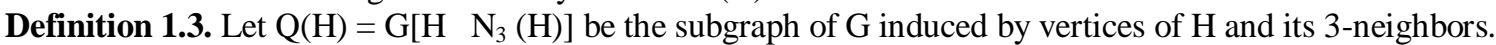

For a path $S=v_{0} v_{1} \ldots v_{m}$, and two vertices $v_{i}, v_{j}$ of $S$ we write $v_{i} S v_{j}$ to denote the subpath $v_{i} v_{i} \ldots v_{j-1} v_{j}$ of $S$. Many of the results presented here will deal with graphs that can be drawn in the plane so that no edges cross.

Definition 1.4. An embedding of a graph $G=(V, E)$ is a map onto $R^{2}$ such that

1. vertices are distinct,

2. edges are paths between the vertices, and

3. edges only intersect at vertices.

A graph is planar if it can be embedded in the plane.

Definition 1.5. A plane graph is a fixed embedding of a (planar) graph where the arcs representing the edges do not intersect other points of the embedding except at the endpoints.

Definition 1.6. A face $f$ of a plane graph $G$ is a connected component of $R^{2}-G$, the regions in the plane that are not covered by the plane graph $G$. The boundary of a face $f$ are the edges and vertices that separate $f$ from the other faces of $\mathrm{G}$.

Definition 1.7. A graph is a triangulation if every face has three edges on its boundary. A graph is a near-triangulation if the boundary of every face except the outer face has three edges on its boundary.

Definition1.8. An graph is outerplanar if it is a planar graph that can be embedded in the plane so that all of the vertices belong to the boundary of the unbounded face. An outerplane graph is such an 
embedding of an outerplanar graph.

Definition 1.9. In a cycle $C$, a chord is an edge between two nonadjacent vertices of the chord which itself is not an edge of the cycle. In this dissertation, we will use the term chord when referring to an edge between two nonadjacent vertices that lie on the cycle that corresponds to the boundary of the unbounded face of a plane graph G.

Definition1.10. Let $G=(V, E)$ be a planar graph. The dual of $G$ is the graph $G=\left(V^{\prime}, E^{\prime}\right)$ whose vertex set $V$ is made up of vertices $v$, each of which corresponds to exactly one plane region of $G$. The edge set $E$ of $G$ is made up of edges e , each of which exists if and only if there is a corresponding edge e joining two adjacent regions in a fixed plane embedding of $\mathrm{G}$. Note that dual graphs are not unique because they depend on the particular embedding used. In this dissertation, we will refer to the notion of a weak dual.

Definition 1.11. The weak dual of $G$ is the induced subgraph $G=(V, E)$ of the dual $G$ whose vertices $v$ correspond to the bounded faces of G. It can be observed that the dual and weak dual of a graph will not necessarily be a simple graph.

Definition 1.12. The triple $\left(x_{1}, x_{2}, x_{3}\right)$ is a triangle in $G$ if $x_{1}, x_{2}, x_{3} \in V(G)$ and $x_{1} x_{2}, x_{2} x_{3}, x_{1} x_{3} \in E(G)$.

Definition 1.13. Let $G=(V, E)$ be a near-triangulation. Assume the non-triangular face is the cycle $C=v_{1} v_{2} \ldots$ $\mathrm{v}_{\mathrm{k}} \mathrm{v}_{1}$. Fix vertices $\mathrm{v}_{1}, \mathrm{v}_{2}, \mathrm{v}_{\mathrm{k}}$.

1. If $\mathrm{V}=\mathrm{V}(\mathrm{C}) \quad\{\mathrm{v}\}$ and $\mathrm{E}=\mathrm{E}(\mathrm{C}) \quad\left\{\mathrm{vv}_{1}, \mathrm{vv}_{2}, \ldots, \mathrm{vv}_{\mathrm{k}}\right\}$, then $\mathrm{G}$ is said to be a wheel $\mathrm{W}$ with center $\mathrm{v}$.

2. If $\mathrm{V}=\mathrm{V}(\mathrm{C})$ and $\mathrm{E}=\mathrm{E}(\mathrm{C}) \quad\left\{\mathrm{v}_{1} \mathrm{v}_{3}, \mathrm{v}_{1} \mathrm{v}_{4}, \ldots, \mathrm{vv}_{\mathrm{k}}\right\}$, then $\mathrm{G}$ is said to be a broken wheel $\mathrm{BW} \mathrm{k}_{\mathrm{1}}$. (This graph is also referred to as a fan $\mathrm{F}_{\mathrm{k}-1}$.)

3. A generalized wheel is a graph that is either a wheel, broken wheel, or one of the following two types of graphs:

(a) $\mathrm{V}=\mathrm{V}(\mathrm{C}) \cup\{\mathrm{u}, \mathrm{v}\}$ and $\mathrm{E}=\mathrm{E}(\mathrm{C})\left\{\mathrm{v}_{1} \mathrm{v}_{\mathrm{i}}, \mathrm{vv}_{\mathrm{i}}, \mathrm{vv}_{\mathrm{i}}, \ldots, \mathrm{vv}_{\mathrm{k}}, \mathrm{uv}_{\mathrm{k}} \mathrm{uv}_{1}, \ldots, \mathrm{uv}_{\mathrm{i}}\right\}$, or

(b) $\mathrm{V}=\mathrm{V}(\mathrm{C}) \cup\{\mathrm{v}\}$ and $\mathrm{E}=\mathrm{E}(\mathrm{C})\left\{\mathrm{v}_{1} \mathrm{v}_{3}, \mathrm{v}_{1} \mathrm{v}_{4}, \ldots, \mathrm{v}_{1} \mathrm{v}_{\mathrm{k}}, \mathrm{vv}_{\mathrm{i}}, \mathrm{vv}_{\mathrm{i}},+1 \ldots, \mathrm{vv}_{\mathrm{k}}\right\}$.

For a generalized wheel, $\mathrm{v}_{\mathrm{k}}, \mathrm{v}_{1} \mathrm{v}_{2}$ is the principal path, $\mathrm{v}_{1}$ is the major vertex, and the edges $\mathrm{v}_{1} \mathrm{v}_{2}, \mathrm{v}_{1} \mathrm{v}_{\mathrm{k}}$ are principal edges. See Figure 2.1 for an illustration of each of the types of generalized wheels described above.

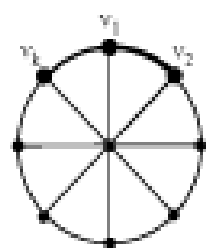

(a) Wheed

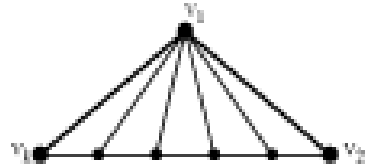

(b) Broken wheal

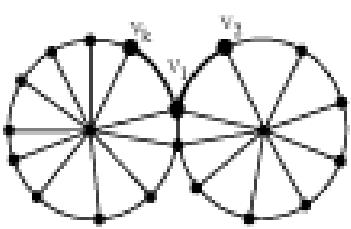

(c)

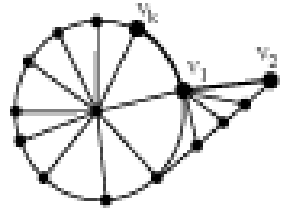

(d)

Figure 2.1: Examples of generalized wheels.

Definition 1.14. The Cartesian product $G \times H$ is the graph with vertex set $V(G) \times V(H)$ where any two vertices $\left(\mathrm{u}, \mathrm{u}^{\prime}\right)$ and (v, v' ) are adjacent in $\mathrm{G} \times \mathrm{H}$ if and only if either $\mathrm{u}^{\prime}=\mathrm{v}^{\prime}$ and $\mathrm{u}$ is adjacent to $\mathrm{v}$ in $\mathrm{H}$, or $\mathrm{u}=\mathrm{v}$ and $\mathrm{u}$ is adjacent to $\mathrm{v}$ in $\mathrm{G}$.

Definition 1.15. The theta graph $\Theta \mathrm{k}_{1}, \mathrm{k}_{2}, \mathrm{k}_{3}$ is the union of three internally disjoint paths with $\mathrm{k}_{1}, \mathrm{k}_{2}, \mathrm{k}_{3}$ internal vertices, respectively, that have the same two distinct end vertices.

Definition 1.16. The graph $G^{k}$, the kth power of a graph $G$, is the graph with the same k vertex set as $G$ and an edge between vertices $u$ and $v$ if and only if there is a path of length at most $k$ between $u$ and $v$ in $G$.

We define the following graph that is obtained by laying cycles of arbitrary and varying lengths greater than 3 end to end so that they share an edge.

Definition 1.17. A graph $G$ is called a path of $k$ cycles, or path of cycles, if $G=\bigcap_{i=1}^{k} G_{i}$ where each $G$ is a cycle of length $\mathrm{a}_{\mathrm{i}} \geq 4$ for $\mathrm{i}=1, \ldots, \mathrm{k}, \mathrm{V}\left(\mathrm{G}_{\mathrm{i}=1}\right) \cap \mathrm{V}\left(\mathrm{G}_{\mathrm{i}}\right)=\left\{\mathrm{t}_{\mathrm{i}}, \mathrm{b}_{\mathrm{i}}\right\}^{\mathrm{i}=1}$ and $\mathrm{E}\left(\mathrm{G}_{\mathrm{i}-1}\right) \cap \mathrm{E}\left(\mathrm{G}_{\mathrm{i}}\right)=\left\{\mathrm{t}_{\mathrm{i}}, \mathrm{b}_{\mathrm{i}}\right\}$ for all $\mathrm{i}=2, \ldots, \mathrm{k}$, and $\mathrm{t}_{\mathrm{i}}, \mathrm{b}_{\mathrm{i}} \notin \mathrm{V}\left(\mathrm{G}_{\mathrm{j}}\right)$ for al $\mathrm{l} \mathrm{j} \neq \mathrm{i}-1$, i. If

$\mathrm{W}_{\mathrm{i}} \in \mathrm{V}\left(\mathrm{G}_{\mathrm{i}}\right)-\left\{\mathrm{t}_{\mathrm{i}}, \mathrm{b}_{\mathrm{i}}, \mathrm{t}_{\mathrm{i}+1}, \mathrm{~b}_{\mathrm{i}+1}\right\}$, then $\mathrm{w}_{\mathrm{i}} \notin \mathrm{V}\left(\mathrm{G}_{\mathrm{j}}\right)$ for all $\mathrm{j} \neq \mathrm{i}$. Furthermore, $\mathrm{G}$ can be drawnin the plane so that the weak dual of $G$ is a path.

We also define a graph that is obtained by laying cycles of arbitrary and varying lengths greater than 3 along a special tree-like structure so they share an edge. 


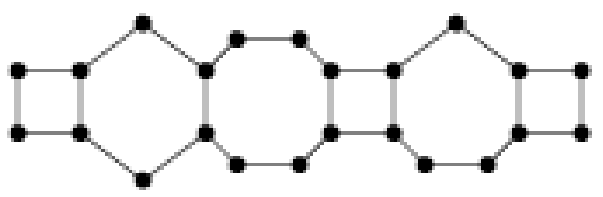

Figure 2.2: An example of a path of cycles.

Definition 1.18. A graph $G$ is called a tree of k cycles, or tree of cycles, if $G=\bigcap_{i=1}^{k} G_{i}$ where each $G$ is a cycle of length $a_{i} \geq 4$ for $i=1, \ldots, k$ and for all pairs $i, j$, it must be that $V\left(G_{i}\right) \cap V\left(G_{j}\right)=\varnothing$ or $V\left(G_{i}\right) \cap V\left(G_{j}\right)=\{u$, $\mathrm{v}\}$ for two adjacent vertices $\mathrm{u}, \mathrm{v} \in \mathrm{V}(\mathrm{G})$. If $\mathrm{V}\left(\mathrm{G}_{\mathrm{i}}\right) \cap \mathrm{V}\left(\mathrm{G}_{\mathrm{j}}\right)=\{\mathrm{u}, \mathrm{v}\}$, then $\mathrm{u}, \notin \mathrm{V}\left(\mathrm{G}_{\mathrm{l}}\right)$ for all $l=\mathrm{i}, \mathrm{j}$. If $\mathrm{w}_{\mathrm{i}}$ $\in \mathrm{V}(\mathrm{G})$, then $\mathrm{w} \notin \mathrm{V}\left(\mathrm{G}_{\mathrm{j}}\right)$ for all $\mathrm{j} \neq \mathrm{i}$ unless $\mathrm{V}\left(\mathrm{G}_{\mathrm{i}}\right) \cap \mathrm{V}\left(\mathrm{G}_{\mathrm{j}}\right)=\{\mathrm{u}, \mathrm{v}\}$ and $\mathrm{w} \quad\{\mathrm{u}, \mathrm{v}\}$. Furthermore, $\mathrm{G}$ can be drawn in the plane so that the weak dual of $G$ is a tree and $G$ can be drawn in the plane so that in the dual of $G$, the vertex corresponding to the unbounded face of $\mathrm{G}$ is adjacent to all other vertices.

See Figure 2.3 for an example of a tree of cycles. Note that a path of cycles is a special case of a tree of cycles that occurs when the underlying tree-like structure is a path.

\section{REDUCTIONS}

When looking at plane graphs, all graphs considered will be assumed to be 2-connected. Assume otherwise, then it is possible to add edges between some pairs of vertices on the boundary of the unbounded face so that the modified graph is 2-connected and all vertices on the boundary of the unbounded face of the original graph are on the boundary of the unbounded face of the modified graph, see Lemma 2.19 below. Any listcoloring of this new graph will then provide a list-coloring of the original graph.

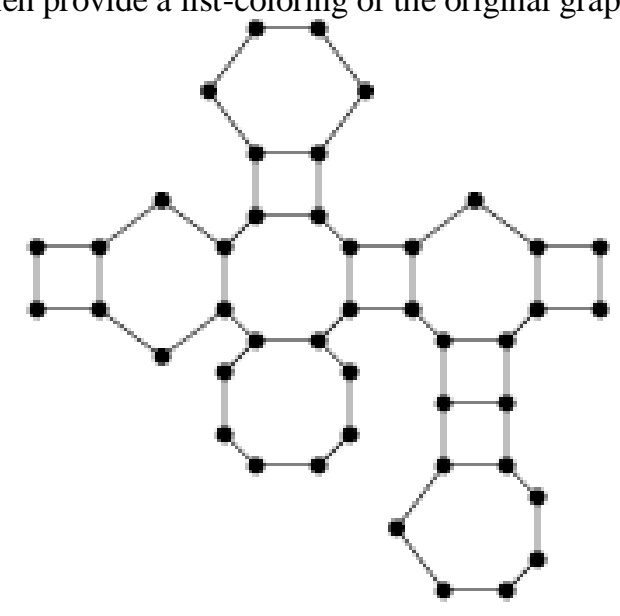

Figure 2.3: An example of a tree of cycles.

Lemma 1.19. Let $G=(V, E)$ be a plane graph that is not 2-connected. Let $F$ be the set of vertices on the boundary of the unbounded face of $\mathrm{G}$. Then it is possible to add edges between some pairs of vertices in F so that the modified graph $G^{\prime}=\left(V^{\prime}, E^{\prime}\right)$ is 2-connected and all vertices of $F$ lie on the boundary of the unbounded face of G'.

Proof. Since G is not 2-connected, there is at least one vertex $v \in V$ that is a cut-vertex of $G$, otherwise $G$ will be disconnected. An edge e must be added to $G$ so that in the resulting graph, call it $G$, the vertex $v$ is not a cut vertex. Additionally, we must add this edge in such a way that all vertices of $\mathrm{F}$ lie on the boundary of the unbounded face of $\mathrm{G}$. Let $\mathrm{W}=\mathrm{vv}_{2}, \ldots \mathrm{v}_{\mathrm{m}} \mathrm{v}$ be a closed walk through all the vertices of $\mathrm{F}$ along the boundary of the unbounded face of G. Since G is not 2-connected, this closed walk is not a cycle and some of the vertices, perhaps edges also, are repeated but not in the same order. In particular, $v$ is repeated. Add the edge $v_{m} v_{2}$ to $G$ so that it now lies on the boundary of the unbounded face of $G$ instead of $v v_{2}$. It is important to note that the order matters here. If $\mathrm{v}_{\mathrm{m}} \mathrm{vv}_{2}$ was part of $\mathrm{W}$, it will also be part of the corresponding closed walk through all of the vertices along the boundary of the unbounded face of $G$. The vertex $v$ is not a cut vertex in the graph $G$. This process may be repeated with each remaining cut vertex so that the modified graph does not contain any cut vertices.

Now that we are assuming all graphs in consideration are 2-connected, we may also employ the following result, see. 
Proposition1.20. Let G be a 2-connected plane graph. Then every edge of G belongs to the boundary of exactly two faces and the boundary of every face is a cycle. In particular, it can be assumed that the boundary of the face corresponding the un-bounded face of $\mathrm{G}$ is a cycle.

In Chapters 3 and 4 we use two different notions of a reduced graph. This allows for us to get information about certain forbidden subgraphs of such graphs. We define the necessary terminology here and then define these reductions. In Chapters 3 and 4 we will show why such reductions can be made.

Definition 1.21. Let $X \subset V$ be a subset of vertices in a connected graph $G=(V, E)$. If $G-X$ contains at least two connected components, then $\mathrm{X}$ is said to be a separating set. If $\mathrm{X}$ is also an i-vertex set spanning an i-cycle, then $\mathrm{X}$ is a separating $\mathrm{i}$-cycle.

Definition 1.22. Let $X \subset V$ be a separating set in a graph $G=(V, E)$. If $P \subset V$ and there are at least two vertices of $\mathrm{P}$ in distinct connected components of $\mathrm{G}-\mathrm{X}$, then $\mathrm{X}$ is a P-separating set. If $\mathrm{X}$ is also a separating $\mathrm{i}$-cycle, then $\mathrm{X}$ is said to be a P-separating i-cycle.

In particular, if $\mathrm{X}$ consists of two adjacent vertices $\mathrm{u}, \mathrm{v}$ and $\mathrm{X}$ is $\mathrm{P}$-separating in $\mathrm{G}$, then $\mathrm{uv}$ is an $\mathrm{P}$-separating edge. If an edge does not have this property, it is called a non-P- separating edge. When looking at a plane graph $\mathrm{G}$, we will use the following:

Definition 1.23. Let $\mathrm{G}$ be a plane graph and let $\mathrm{C}$ be the cycle that corresponds to the boundary of the unbounded face of G. If the edge uv is a chord in $\mathrm{G}$ with endpoints in $\mathrm{C}$ and a $\mathrm{P}$ - separating edge, then uv is called a $\mathrm{P}$ separating chord.

Definition 1.24. We say that a set $X$ of four vertices of degree at most 5 in a graph $G$ forms the configuration $D=$ $\mathrm{D}(\mathrm{X})$ if $\mathrm{G}[\mathrm{X}]$ is isomorphic to $\mathrm{K}_{4-\mathrm{e}}$.

See Figure 2.4a for an illustration of D.

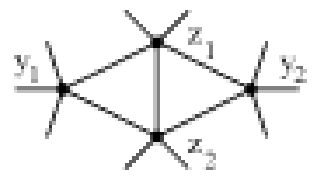

(a) Configuration $D$

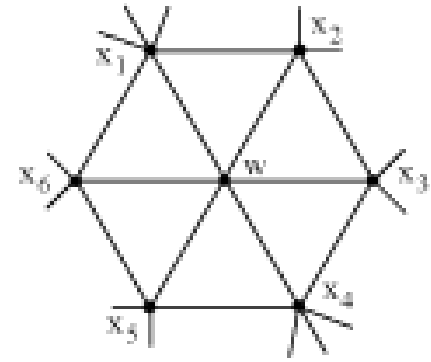

(b) Configuration $W$.

Figure 2.4: Reducible configurations.

Definition 1.25. We say that a set $X$ of seven vertices of degree at most 6 in a graph $G$ forms the configuration $W$ $=\mathrm{W}(\mathrm{X})$ if $\mathrm{G}[\mathrm{X}]$ induces a 6-wheel, formed from a central vertex w adjacent to a 6-cycle $\mathrm{x}_{1} \mathrm{x}_{2} \mathrm{x}_{3} \mathrm{x}_{4} \mathrm{X}_{5} \mathrm{x}_{6} \mathrm{x}_{1}$ such that $\mathrm{x}_{2}, \mathrm{x}_{3}, \mathrm{x}_{5}$ and $\mathrm{x}_{6}$ have degree at most 5 in G. See Figure $2.4 \mathrm{~b}$ for an illustration of W.

We now have all of the terminology needed to define the two types of reductions that will be used in this thesis. The first type will be used in Chapter 3 and the second type will be used in Chapter 4 .

Definition 1.26. For a graph $G$ and a set of vertices $P$, let $R(G)=R(G, P)$, a Type I reduction of $G$ with respect to $\mathrm{P}$, be a graph obtained by performing one of the following operations on $\mathrm{G}$ :

1. for a separating 3-cycle or 4-cycle that does not separate $P$, remove from $G$ the vertices and edges in the region that is bounded by the separating 3-cycle or 4-cycle and that does not contain any vertices of $\mathrm{P}$,

2. for a configuration $D=D(X)$ such that $P n X=\emptyset$, remove $X$ from $G$, or

3. for a configuration $W=W(X)$ such that $P n X=\varnothing$, remove $X$ from $G$.

If none of these operations can be carried out, let $R(G)=G$.

Consider a sequence of graphs $\mathrm{G}=\mathrm{G}_{0} \supset \mathrm{G}_{1} \supset \mathrm{G}_{2} \supset \cdots \supset \mathrm{G}_{\mathrm{m}}$ such that $\mathrm{G}_{\mathrm{i}}=\mathrm{R}\left(\mathrm{G}_{\mathrm{i}-1}, \mathrm{P}\right)$

for $\mathrm{i}=1, \ldots, \mathrm{m}$ and $\mathrm{R}\left(\mathrm{G}_{\mathrm{m}}\right)=\mathrm{G}_{\mathrm{m}}$. Call such a graph $\mathrm{G}_{\mathrm{m}}$ a Type I reduced graph of $\mathrm{G}$. A Type I reduced graph does not have a separating 3-cycle or 4-cycle that does not separate $\mathrm{P}$ and it contains no configurations $\mathrm{D}(\mathrm{X})$ or $\mathrm{W}(\mathrm{X})$ with $P \cap X=\varnothing$. We shall show in Chapter 3 that if a Type I reduced graph of $G$ has a coloring extension of $P$, then so does $\mathrm{G}$.

Definition 1.27. Let $\mathrm{G}$ be a plane graph and let $\mathrm{C}$ be the cycle that corresponds to the boundary of the unbounded face of $G$. Let $x, y \in V(C)$. Let $R(G)=R(G,\{x, y\})$, a Type II reduction of $G$ with respect to $x$, $y$, be a graph obtained by performing one of the following operations on $\mathrm{G}$ :

1. If $X$ is a set of vertices in $G$ that induces a separating 3-cycle or a separating 4-cycle and $X^{1}$ is the vertex set of the connected component of $G-X$ which contains neither $x$ nor $y$, then let $R(G)=G-X$.

2. If there is a non- $\{x, y\}$-separating chord $u v$ in $G$ that splits $G$ into two graphs $G_{A}$ and $G_{B}$ such that $G=G_{A}$ 
$\cup \mathrm{G}_{\mathrm{B}}, \mathrm{V}\left(\mathrm{G}_{\mathrm{A}}\right) \cap \mathrm{V}\left(\mathrm{G}_{\mathrm{B}}\right)=\{\mathrm{u}, \mathrm{v}\}$, and $\mathrm{x}, \mathrm{y} \in \mathrm{V}\left(\mathrm{G}_{\mathrm{A}}\right)$, then let $\mathrm{R}(\mathrm{G})=\mathrm{G}_{\mathrm{A}}$.

If neither of these operations can be carried out, then $R(G)=G$.

Consider a sequence of graphs $G=G_{0} \supset G_{1} \supset G_{2} \supset \cdots \supset G_{m}$ such that $G_{i}=R\left(G_{i-1},\{x, y\}\right)$ for $i=1, \ldots, m$ and $R\left(G_{m}\right)=G_{m}$. Call such a graph $G_{m}$ a Type II reduced graph of G. Note that a Type II reduced graph does not contain any separating 3-cycles, separating 4-cycles, nor any non- $\{\mathrm{x}, \mathrm{y}\}$-separating chords. Observe also that if $G$ is 2-connected, then $R(G)$ is 2-connected.

Let $\mathrm{f}^{\mathrm{G}-\mathrm{H}}(\mathrm{v})=\mathrm{f}(\mathrm{v})-\mathrm{d}(\mathrm{v}, \mathrm{G}-\mathrm{H})$ for all $\mathrm{v} \in \mathrm{V}(\mathrm{G}-\mathrm{H})$. The subgraph $\mathrm{H}$ is called $\mathrm{G}-\mathrm{H}$

a reducible configuration with respect to $\mathrm{f}$ if $\mathrm{H}$ is $\mathrm{f}^{\mathrm{G}-\mathrm{H}}$-choosable. We say that $\mathrm{H}$ is reducible because if $\mathrm{H}$ satisfies these conditions and $\mathrm{G}-\mathrm{H}$ is $\mathrm{f}_{\mathrm{G}-\mathrm{H}}$-choosable, then $\mathrm{G}$ will be $\mathrm{f}$-choosable. Thus, to show that $\mathrm{G}$ is $\mathrm{f}$ choosable, we can reduce the problem to showing that $\mathrm{G}-\mathrm{H}$ is $\mathrm{f}_{\mathrm{G}-\mathrm{H}}$-choosable for any such $\mathrm{H}$. In many cases, this will simplify the work that needs to be done. This is because it allows for the assumption that no such reducible configuration exists as a subgraph. Such an assumption will often lead to a contradiction. The good configurations of Chapter 5 can be considered reducible configurations as subgraphs of a planar graph with 5-lists assigned to its vertices.

\section{CONCLUSION}

We discussed some results on 2-connected.

\section{REFERENCES}

[1] Dimitris Achlioptas and Cristopher Moore. Almost all graphs with average degree 4 are 3-colorable. J. Comput. System Sci., 67(2):441\{471, 2003. Special issue on STOC2002 (Montreal, QC).

[2] Michael O. Albertson. You can't paint yourself into a corner. J. Combin. Theory Ser. B, 73(2):189\{194, 1998.

[3] Michael O. Albertson, Alexandr V. Kostochka, and Douglas B. West. Precoloring extensions of Brooks' theorem. SIAM J. Discrete Math., 18(3):542\{553, 2004/05.

[4] Noga Alon. Restricted colorings of graphs. In Surveys in combinatorics, 1993 (Keele), volume 187 of London Math. Soc. Lecture Note Ser., pages 1\{33. Cambridge Univ. Press, Cambridge, 1993.

[5] Noga Alon. Degrees and choice numbers. Random Structures Algorithms, 16(4):364\{368, 2000.

[6] Kenneth Appel and Wolfgang Haken. Every planar map is four colorable. Bull. Amer. Math. Soc., 82(5):711\{712, 1976. 